\title{
"More Air - Better Performance - Faster Recovery": Study Protocol for Randomised Controlled Trial of the Effect of Post-stroke Inspiratory Muscle Training for Adults
}

\section{Susanne Lillelund Sørensen}

Regional Hospital Hammel Neurocenter: Regionshospitalet Hammel Neurocenter https://orcid.org/0000-0002-1315-3546

\section{Simon Svanborg Kjeldsen}

Regional Hospital Hammel Neurocenter: Regionshospitalet Hammel Neurocenter Sine Secher Mortensen

Regional Hospital Hammel Neurocenter: Regionshospitalet Hammel Neurocenter Ulla Torp Hansen

Regional Hospital Hammel Neurocenter: Regionshospitalet Hammel Neurocenter Dorthe Hansen

Regional Hospital Hammel Neurocenter: Regionshospitalet Hammel Neurocenter

\section{Asger Roger Pedersen}

Regional Hospital Hammel Neurocenter: Regionshospitalet Hammel Neurocenter

Hanne Pallesen ( $\boldsymbol{Q}$ hannpall@rm.dk)

Regional Hospital Hammel Neurocenter: Regionshospitalet Hammel Neurocenter

\section{Study protocol}

Keywords: Inspiratory muscle training, fatigue, stroke, endurance in gait, voice volume and phonation, activity of daily living, spirometry, peak expiratory flow

Posted Date: June 9th, 2021

DOI: https://doi.org/10.21203/rs.3.rs-587798/v1

License: (c) (1) This work is licensed under a Creative Commons Attribution 4.0 International License. Read Full License 


\section{Abstract}

Background: Stroke results in varying physical, cognitive, emotional and/or social disabilities in the short and long term alike. Motor impairments are important, persistent consequences of stroke and include, among others, decreased respiratory muscle function, decreased ability to expand the thorax and postural dysfunction. These deficits affect the patient's ability to perform daily activities, produce fatigue and reduce endurance and quality of life. Inspiratory muscle training (IMT) aims to improve the strength and endurance of the diaphragm and the external intercostal muscles. The objective of this study was to investigate i) the effect of three weeks of IMT on maximal inspiratory pressure (MIP) in adults having suffered a stroke, and ii) the effects of three weeks of IMT on the degree of dependency in activities of daily living, endurance in gait, fatigue, voice volume, phonation endurance and expiratory function.

Methods/Design: This is a randomised controlled trial (RCT) comparing IMT with conventional neurorehabilitation (usual practise). The trial will include 80 patients with reduced MIP hospitalised at a specialised neurorehabilitation hospital in Denmark. The intervention group will receive IMT sessions, exercising at $30 \%$ of MIP. Patients in the intervention group will perform two daily sessions (one session of IMT with Threshold IMT consisting of two times 15 inspirations at normal breathing rhythm (5-10 $\min )$ ), seven days a week for three weeks. Training can be with or without physiotherapist supervision. Study outcomes: MIP assessed by the Power Breath, Functional Independence Measurement, The 6minute Walk Test, the Fatigue Severity Scale and average voice volume. Expiratory function will be assessed using spirometry. All assessments will be conducted at baseline and three weeks (at termination of the intervention) and three months after the intervention has concluded.

Discussion: The primary purpose of this study is to assess the effect of IMT interventions in patients with reduced MIP following stroke; secondarily, to assess the effect of IMT on the degree of dependency in activities of daily living, endurance in gait, fatigue, voice volume, phonation endurance and expiratory capacity. Hereby, this study accepts the call for further research.

Trial registration: ClinicalTrials.gov NCT04686019. Registered 28.12.2020, https://www.clinicaltrials.gov/ct2/show/NCT04686019

\section{Background}

In Denmark (counting approximately 5.8 million inhabitants (1)), 12,500 people experience stroke annually (2). The incidence is highest in the elder part of the population. As the average life expectancy increases, stroke is expected to become an even heavier burden on the healthcare system $(2,3)$. Stroke results in varying physical, cognitive, emotional and/or social disabilities in the short and long term alike $(4,5)$. Motor impairments are significant and persistent consequences of stroke and include decreased respiratory muscle function, decreased ability to expand the thorax and postural dysfunction, among others (6-9). These deficits affect the patient's ability to engage in daily activities, produce fatigue, reduce endurance and affect quality of life. 
Inspiratory muscle training (IMT) aims to improve the strength and endurance of the diaphragm and external intercostal muscles (9). In sports, IMT serves to improve athletes' respiratory capacity $(10,11)$. In Denmark, a few IMT tests have been conducted with positive results, for instance in persons with tetraplegia in the acute phase. IMT has also been used successfully in people with COVID-19; however, apart from these areas, knowledge of the use of IMT is surprisingly sparse. This project will be one of the first to bridge this knowledge gap.

Smaller studies have tested the method in post-stroke patients, and results indicate that IMT has a positive effect on respiratory muscle function, exercise capacity and quality of life (12-14). A review recommends using IMT as an intervention for patients suffering from chronic obstructive pulmonary disease (COPD) (15). IMT is less known and less frequently used in neurorehabilitation, but clinical experiences imply that IMT may improve voice volume, phonation endurance and coughing force (airway clearance). It would be useful to substantiate evidence presented for this hypothesis. It is therefore relevant to test the intervention in a larger study setup.

The objective of this study was to investigate:

- the effect of three weeks of IMT on maximal inspiratory pressure (MIP) in adults having suffered stroke.

- the effects of three weeks of IMT on the degree of dependency in activities of daily living, endurance in gait, fatigue, voice volume (VV), phonation endurance (PE) and expiratory function; peak expiratory flow (PEF), forced expiratory volume in 1 second (FEV1), forced vital capacity (FVC) and FEV1/FVC ratio.

\section{Methods/design}

\section{Design}

This study is a randomised controlled trial (RCT) comparing IMT with conventional neurorehabilitation (usual practice). Baseline assessments are made 0-4 days before the intervention starts. Further assessments are performed 0-4 days after the intervention concludes and at the three-month follow-up. Assessments will be conducted by assessors blinded to the randomisation and the intervention.

\section{Patient population}

We plan to enrol 80 patients in the study. All patients are hospitalised at the specialised neurorehabilitation hospital in Denmark. Inclusion criteria are:

- First-time brain infarction or brain haemorrhage within the past 0-3 months

- Age $>17$ years

- Able to give written consent and being cognitively and communicatively able to understand and participate in the MIP test 
- Reduced MIP below the gender- and age-specific normal standard given in Table 1

Table 1: MIP - gender- and age-specific standard

\begin{tabular}{|c|c|c|c|c|c|}
\hline \multicolumn{6}{|c|}{ Normalvardier for Maximal inspiratorisk Tryk (PL-w) } \\
\hline Alder & $20-54$ & 55.59 & 60.64 & $65 \cdot 69$ & $70-74$ \\
\hline Mand & $124 \pm 44$ & $103 \pm 32$ & $103 \leq 32$ & $103 \pm 32$ & $103+32$ \\
\hline Kvinder & $87 \pm 32$ & $77 \pm 26$ & $73 \geq 26$ & $70 \pm 26$ & $65 \pm 26$ \\
\hline
\end{tabular}

Patients with the following characteristics will be excluded: diagnosis of myocardial infarction within the past three months, significant pulmonary disease (severe COPD), saturation below 90 if having COPD and for others a saturation below 92, neurological deficits other than stroke, facial palsy affecting proper labial occlusion, dizziness or nausea/vomiting during MIP testing.

\section{Randomisation}

An independent, centralised randomisation database (RedCap) will provide allocation concealed to involved clinicians and assessors. A stratified block randomisation of severity of impairment based on Modified Rankin Scale (mRS) $(<4)$ and block size of 4 with an intervention/control ratio of $1 / 3$ will be performed. Furthermore a stratified block randomisation of the two participating neurorehabilitation units will be performed.

\section{Intervention}

Participants in both groups will receive conventional neurorehabilitation - treatment as usual. The intervention group will receive add-on IMT sessions with exercising at $30 \%$ of MIP. MIP is measured by instructing the patient to perform five forceful inspirations against an occluded mouthpiece Power Breathe KH2 (POWERbreathe International Ltd, UK). The best score is multiplied by 0.3 to determine resistance. IMT is performed with the Threshold IMT (Philips, UK) with the calculated resistance. During the Power Breath measurement, the patient sits straight up and uses a nose clip. The patient may receive help to hold the Power Breath if necessary.

One session of IMT with the Threshold IMT consists of two times 15 inspirations at normal breathing rhythm (5-10 min). Patients in the intervention group perform two sessions a day (morning and evening), training is performed prior to a meal, seven days a week for three weeks. According to the patients' need of support, IMT can be with or without supervision. MIP is reassessed every seven days, and a new resistance is calculated. Patients in the intervention group log their IMT in a log book. The log book supports the patient in performing the right number of respirations twice a day for three weeks. The patient sits straight up in a chair/wheelchair/armchair, possibly with a table in front of him or her during practice. The Threshold IMT mouthpiece is placed after the resistance has been set manually. The nose clip must be used during practice to ensure that air passes through the mouth only.

\section{Outcomes}


The primary outcome measure is MIP assessed by Power Breath (16). This is an objective measurement used to describe the inspiratory capacity.

Secondary outcome measures include: the Functional Independence Measure (FIM) to assess the degree of dependency in daily activities - both total score and motor sub score (17). The 6-minute walking test to measure endurance (18-20). Fatigue measured by the Fatigue Severity Scale $(21,22)$. Average voice volume will be measured by recording of an a-sound with the app Voice Analyst. Phonation endurance (how long the a-sound lasts) is noted. The patient tries three times and the average is then calculated. Expiratory function: peak expiratory flow (PEF), forced expiratory volume in 1 second (FEV1), forced vital capacity (FVC) and FEV1/FVC ratio will be assessed with Spirometry device (Vitalograph Micro 6300, Ireland). All respiratory measures are performed in sitting position. The patient tries three times. Subsequently, the best score is used for further analysis (12).

All assessments will be conducted at baseline, three weeks (at termination of the intervention) and three months after the intervention concluded. The Patient Global Impression of Change (PGIC) scale will be employed upon conclusion of the intervention (23).

\section{Test Procedure}

Tests and the questionnaires are given and completed at three time points: at baseline, at three weeks and at three months after the intervention has concluded. Outcome assessors are blinded to the intervention. Testing takes place in a specific room at the recruitment hospital. Outcome assessors are physiotherapists trained in performing the test procedures and are not joining the treatment team at the recruiting ward. Patients will be tested by the same therapist all three times. The trial participants and intervention providers are not blinded.

\section{Sample size}

Power was calculated to establish the needed number of participants. The power calculation was based partly on a local pre-study (including 26 patients) and on a RCT study (12), which showed differences in variance in $\triangle$ MIP assessments. $\triangle$ MIP is defined as (MIP at three months less MIP at baseline). Because of the difference in variances in the intervention group and the control group, the same power can be achieved by distributing them unequally between the groups. It is assumed that the standard deviation is three times larger in the intervention group than in the control group. The power calculation prescribes 20 patients in the control group and 60 patients in the intervention group. Calculations were made, expecting a $10 \%$ dropout. The least clinically relevant difference was estimated to $5 \mathrm{cmH} 2 \mathrm{O}$.

\section{Statistical analysis}

Data are analysed as intention-to-treat and per protocol. Between group differences (three-month followup less baseline) are compared by the T-test. Regression analysis are conducted with adjustment for the confounders age and time from stroke to baseline assessment. 


\section{Study organization}

This study is organised and coordinated by the research unit at the Hammel Neurorehabilitation Centre. The participating stakeholders in this study are clinicians and researchers at the Hammel Neurorehabilitation Centre. All parties participate in the Steering Committee and are involved in discussing interim results and making the final decisions about the trial.

\section{Discussion}

The primary purpose of this study is to assess the effect of an IMT intervention in patients with reduced MIP following stroke. A secondary purpose is to assess the effect of IMT on the degree of dependency in activities of daily living, endurance in gait, fatigue, voice volume, phonation endurance and expiratory capacity. Hereby, this study accepts the call for further research made by Xiao (9).

The study assumes that by strengthening the diaphragm muscle and the intercostal muscles, patients will gain faster respiratory recovery, which, in turn, will allow them to practice or carry out activities of daily living earlier in the course of their rehabilitation. The typical length of IMT interventions is 6-8 weeks $(12,13,24)$. In 2019, the average hospitalisation in the study setting for stroke patients was 34 days ( $95 \% \mathrm{Cl}: 21-52$ days). The length of the intervention was set to 21 days to ensure that the intervention could be completed during hospitalisation. The duration of the intervention was also informed by positive results from the pre-study. We argue that it is interesting to investigate whether or not a shorter intervention period is sufficient for patients to experience a positive impact on MIP and the degree of their dependency in activities of daily living, endurance in gait, fatigue, voice volume, phonation endurance and expiratory function. Especially, we find it valuable to investigate the effect of increasing intensity in rehabilitation as the intervention is relative easy and inexpensive to implement and may be practiced by many patients on their own as an add-on to their rehabilitation.

A broad spectrum of patients with sensorimotor deficits following stroke is expected to be included in the study, as it does not exclude patients based on the severity of their stroke. We find it important to develop interventions that may also have an impact on the more severely injured patients.

Sham IMT was considered for the control group, but was rejected for ethical reasons, as it would be time consuming for patients to do the sham intervention, which would be unethical as these patients need to economise their resources due to fatigue.

An unpublished pre-study without a control group made in one part of the setting of this study indicated improvement in voice volume and phonation endurance not previously described as outcomes associated with IMT. Some patients in the pre-study described that these favourable outcomes enabled them to have phone conversations or longer face-to-face conversations with their relatives, giving them greater quality of life. We expect to obtain further knowledge on this previously undescribed field. 
The pre-study has provided useful information about how to conduct the intervention, and the assessment points were adjusted according to what proved feasible in the pre-study.

\section{Trial status}

Enrolment of participants starts on 01 February 2021. Recruitment, follow-up assessments and data analyses are expected to be completed by the end of December 2022. See Template Fig. 2 and the Trials populated SPIRIT checklist (Additional file 1).

Protocol version 1.1 (December 18, 2020)

\section{Abbreviations}

EF: Expiratory function, FFS: Fatigue Severity Scale, FIM: Functional Independence Measure, IMT: Inspiratory muscle training, mRS: Modified Rankin Scale, MIP: Maximal inspiratory pressure, RCT: Randomised controlled trial, VV\&PE: Voice volume and Phonation endurance.

\section{Declarations}

\section{Ethics approval and consent to participate}

This study was approved by the National Committee on Health Research Ethics in Denmark (Case no. 110-72-237-19) and by the Danish Data Protection Agency. The project complies with the Data Protection Regulation and the Data Protection Act.

Previous studies have shown that the participants are able to conduct the intervention without discomfort. Patients may experience a light respiratory effort. Written informed consent to participate will be obtained, and the study will be conducted in accordance with the Helsinki Declaration of 2008. The trial is registered at ClinicalTrials.gov (NCT04686019). The data are safely stored in a REDCap database hosted by Aarhus University $(25,26)$. REDCap is a secure web application for building and managing online surveys and databases.

\section{Availability of data and materials}

During the data collection period for this study, the researchers and the assessors have access to the REDCap database. The assessors however, have no access to information in regard to randomisation. The REDCap database inform the assessors about data completion. The data collected in this study will be available from the principal investigator on reasonable request.

\section{Consent for publication}

Not applicable.

\section{Competing interests}


The authors declare that they have no competing interests.

\section{Funding}

The trial is partly funded by The Helse Foundation and Regional Hospital Central Jutland Research Fund. The funder has no influence on the design of the protocol and will not have any control of data generation, statistical analyses or writing of the final manuscript. However, a communication agreement concerning the trials results has been approved.

\section{Authors' contributions}

Hanne Pallesen and Susanne Lillelund Sørensen drafted the manuscript. All authors participated in developing the design of the study, contributed to and critically appraised the manuscript. All authors have read and acknowledged the final manuscript.

\section{Acknowledgements}

We are grateful for the financial funding from the Helse Foundation, Regional Hospital Central Jutland Research Fund and the support received from the clinicians working at the participating hospitals/centres.

\section{References}

1. Larsen D. Population in Denmark [Internet]. Copenhagen: Statistics Denmark; 2020 [cited 2020 dec 8]. Available from: www.dst.dk/da/Statistik/emner/befolkning-og-valg/befolkning-ogbefolkningsfremskrivning/folketal.

2. Flachs EM, Statens Institut for F, Sundhedsstyrelsen S. Sygdomsbyrden i Danmark : sygdomme. Kbh.: Sundhedsstyrelsen; 2015. 382 p.

3. Feigin VL, Forouzanfar MH, Krishnamurthi R, Mensah GA, Connor M, Bennett DA, et al. Global and regional burden of stroke during 1990-2010: findings from the Global Burden of Disease Study 2010. Lancet (London, England). 2014;383(9913):245-54.

4. McKevitt C, Fudge N, Redfern J, Sheldenkar A, Crichton S, Rudd AR, et al. Self-reported long-term needs after stroke. 2011. p. 1398-403.

5. Salter K, Hellings C, Foley N, Teasell R. The experience of living with stroke: a qualitative metasynthesis. Journal of Rehabilitation Medicine. 2008;40(8):595-602.

6. Harik-Khan RI, Wise RA, Fozard JL. Determinants of maximal inspiratory pressure. The Baltimore Longitudinal Study of Aging. American journal of respiratory and critical care medicine. 1998;158(5 Pt 1):1459-64.

7. Lanini B, Bianchi R, Romagnoli I, Coli C, Binazzi B, Gigliotti F, et al. Chest Wall Kinematics in Patients with Hemiplegia. American Journal of Respiratory and Critical Care Medicine. 2003;168(1):109-13. 
8. Ward K, Seymour J, Steier J, Jolley CJ, Polkey MI, Kalra L, et al. Acute ischaemic hemispheric stroke is associated with impairment of reflex in addition to voluntary cough. The European respiratory journal. 2010;36(6):1383-90.

9. Xiao Y, Luo M, Wang J, Luo H. Inspiratory muscle training for the recovery of function after stroke. The Cochrane database of systematic reviews. 2012;(5):CD009360. doi(5):CD009360.

10. Hartz CS, Sindorf MAG, Lopes CR, Batista J, Moreno MA. Effect of Inspiratory Muscle Training on Performance of Handball Athletes. Sciendo; 2018. p. 43-51.

11. Kilding AE, Brown S, McConnell AK. Inspiratory muscle training improves 100 and $200 \mathrm{~m}$ swimming performance. European journal of applied physiology. 2010;108(3):505-11.

12. Sutbeyaz ST, Koseoglu F, Inan L, Coskun $\mathrm{O}$. Respiratory muscle training improves cardiopulmonary function and exercise tolerance in subjects with subacute stroke: a randomized controlled trial. SAGE Publications; 2010. p. 240-50.

13. Britto RRPTP, Rezende NIRPT, Marinho KCPT, Torres JLPT, Parreira VnFPTP, Teixeira-Salmela LFPTP. Inspiratory Muscular Training in Chronic Stroke Survivors: A Randomized Controlled Trial. Archives of Physical Medicine and Rehabilitation. 2011;92(2):184-90.

14. Alvarenga MTM, Menezes KKP, Nascimento LR, Avelino PR, Almeida TLS, Teixeira-Salmela LF. Community-dwelling individuals with stroke, who have inspiratory muscle weakness, report greater dyspnea and worse quality of life. (1473-5660 (Electronic)).

15. Beaumont $M$, Forget $P$, Couturaud $F$, Reychler $G$. Effects of inspiratory muscle training in COPD patients: A systematic review and meta?analysis. Wiley Subscription Services, Inc; 2018. p. 2178-88.

16. Evans JA, Whitelaw WA. The assessment of maximal respiratory mouth pressures in adults. Respiratory care. 2009;54(10):1348-59.

17. Kidd D, Stewart G, Baldry J, Johnson J, Rossiter D, Petruckevitch A, et al. The Functional Independence Measure: A comparative validity and reliability study. Informa UK LtdTaylor \& Francis; 1995. p. 10-4.

18. Fulk GD, Echternach JL, Nof L, O'Sullivan S. Clinometric properties of the six-minute walk test in individuals undergoing rehabilitation poststroke. Physiotherapy theory and practice. 2008;24(3):195204.

19. Harmsen WJ, Ribbers GM, Slaman J, Heijenbrok-Kal MH, Khajeh L, van Kooten F, et al. The six-minute walk test predicts cardiorespiratory fitness in individuals with aneurysmal subarachnoid hemorrhage. Topics in Stroke Rehabilitation. 2017;24(4):250-5.

20. Kosak M, Smith T. Comparison of the 2-, 6-, and 12-minute walk tests in patients with stroke. Superintendent of Documents; 2005. p. 103-7.

21. Lorentzen K, Danielsen MA, Kay SD, Voss A. Validation of the Fatigue Severity Scale in Danish patients with systemic lupus erythematosus. Danish medical journal. 2014;61(4):A4808.

22. Krupp LB, LaRocca NG, Muir-Nash J, Steinberg AD. The Fatigue Severity Scale: Application to Patients With Multiple Sclerosis and Systemic Lupus Erythematosus. Archives of Neurology. 1989;46(10):1121-3. 
23. Shkirkova K, Schuberg S, Balouzian E, Starkman S, Eckstein M, Stratton S, et al. Paramedic Global Impression of Change During Prehospital Evaluation and Transport for Acute Stroke Communitydwelling individuals with stroke, who have inspiratory muscle weakness, report greater dyspnea and worse quality of life. (1524-4628 (Electronic)).

24. Jung J-h, Kim N-s. The effect of progressive high-intensity inspiratory muscle training and fixed highintensity inspiratory muscle training on the asymmetry of diaphragm thickness in stroke patients. Journal of Physical Therapy Science. 2015;27(10):3267-9.

25. Harris PA, Taylor R, Minor BL, Elliott V, Fernandez M, O'Neal L, et al. The REDCap consortium: Building an international community of software platform partners. (1532-0480 (Electronic)).

26. Harris PA, Taylor R Fau - Thielke R, Thielke R Fau - Payne J, Payne J Fau - Gonzalez N, Gonzalez N Fau - Conde JG, Conde JG. Research electronic data capture (REDCap)--a metadata-driven methodology and workflow process for providing translational research informatics support. (15320480 (Electronic)).

\section{Figures}




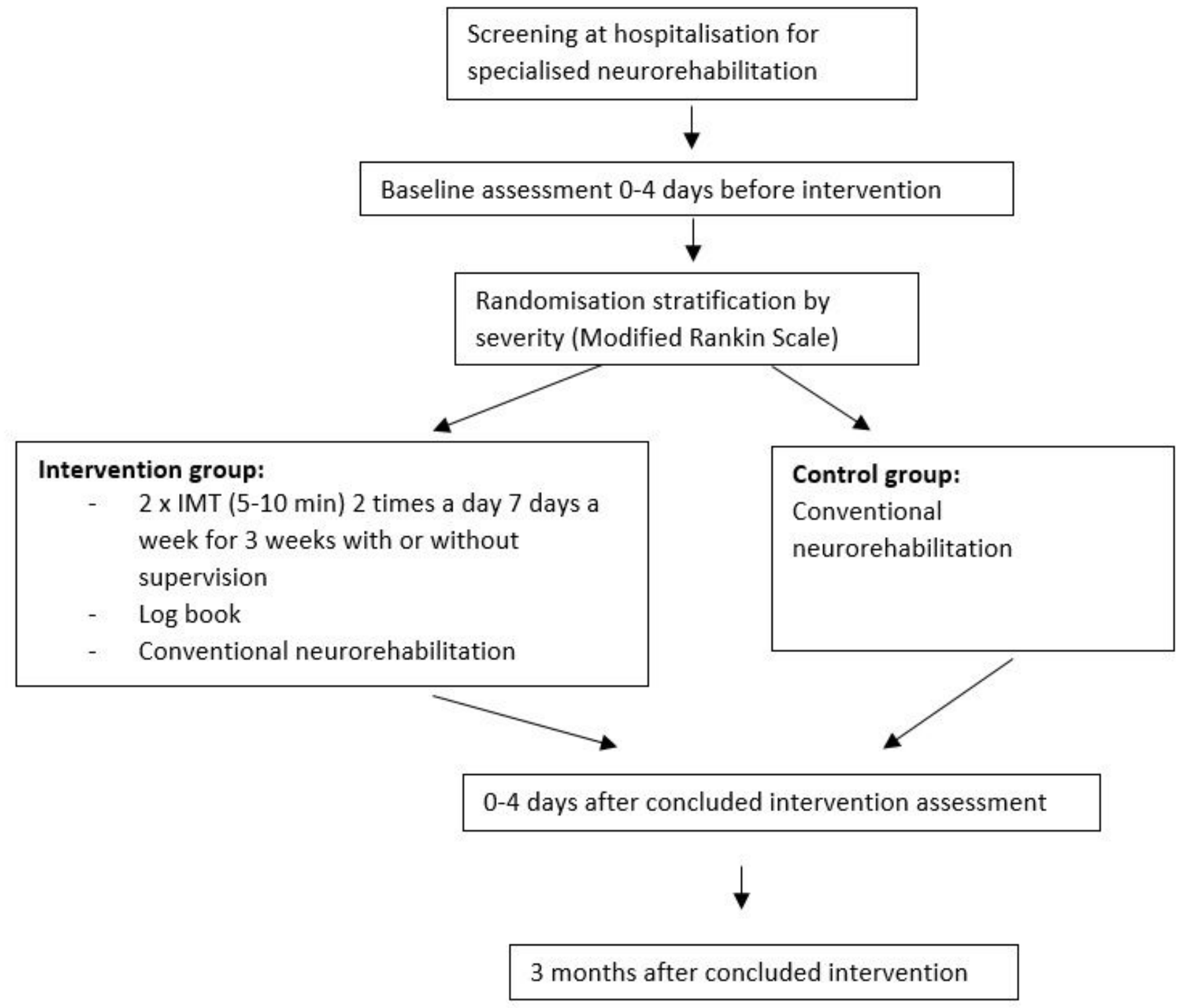

Figure 1

Patient flowchart 


\begin{tabular}{|c|c|c|c|c|c|}
\hline & Enrolment & $\begin{array}{c}\text { All } \\
\text { oca } \\
\text { tio } \\
\text { n }\end{array}$ & & & \\
\hline TIMEPOINT & $-t_{1}$ & 0 & $t_{1}$ & $t_{2}$ & $t_{3}$ \\
\hline ENROLMENT: & $0-3$ months after stroke & & & & \\
\hline Eligibility screen & $\begin{array}{l}\text { First week after hospitalisation } \\
\text { at specialised } \\
\text { neurorehabilitation unit }\end{array}$ & & & & \\
\hline Informed consent & $\begin{array}{l}\text { 2-4 days after hospitalisation at } \\
\text { specialised neurorehabilitation } \\
\text { unit }\end{array}$ & & & & \\
\hline Screening & $\begin{array}{l}\text { 4-6 days after hospitalisation at } \\
\text { specialised neurorehabilitation } \\
\text { unit }\end{array}$ & & & & \\
\hline Randomisation & $\begin{array}{l}\text { 4-6 days after hospitalisation at } \\
\text { specialised neurorehabilitation } \\
\text { unit }\end{array}$ & $\mathrm{x}$ & & & \\
\hline \multicolumn{6}{|l|}{ INTERVENTIONS: } \\
\hline \multirow{2}{*}{$\begin{array}{l}\text { A: IMT } \\
\text { B: Conventional } \\
\text { neurorehabilitation }\end{array}$} & & & $\mathrm{x}$ & $\mathrm{x}$ & \\
\hline & & & $\mathrm{x}$ & $\mathrm{x}$ & \\
\hline \multirow{5}{*}{$\begin{array}{l}\text { ASSESSMENTS: } \\
\text { Baseline: } \\
\text { Inspiratory muscle training } \\
\text { (MIP) } \\
\text { Functional independence } \\
\text { Measure (FIM) } \\
\text { 6-minute walking test } \\
\text { Fatigue Severity Scale (FSS) } \\
\text { Voice volume and phonation } \\
\text { endurance (VV\&PE) } \\
\text { Expiratory function (EF) } \\
\text { 1: Follow-up: } \\
\text { MIP, FIM, 6-minute walking } \\
\text { test, FSS, VV\&PE, EF and } \\
\text { Patient Global Impression of } \\
\text { Change } \\
\text { 2. Second Follow-up } \\
\text { MIP, FIM, 6-minute walking } \\
\text { test, FSS, VV\&PE and EF }\end{array}$} & & & & & \\
\hline & & & $\begin{array}{l}0-4 \text { days } \\
\text { before the } \\
\text { intervention }\end{array}$ & & \\
\hline & & & & $\begin{array}{l}\text { Assessment } \\
0-4 \text { days } \\
\text { after the } \\
\text { intervention } \\
\text { concluded }\end{array}$ & \\
\hline & & & & & $\begin{array}{l}\text { Three } \\
\text { months } \\
\text { after the }\end{array}$ \\
\hline & & & & & $\begin{array}{l}\text { interventio } \\
\mathrm{n} \\
\text { concluded }\end{array}$ \\
\hline
\end{tabular}

Figure 2

Template of recommended contents for schedule of enrolment, interventions and assessments.

\section{Supplementary Files}

This is a list of supplementary files associated with this preprint. Click to download. 
- SPIRITFillablechecklist15Aug2013.doc 